Editorial

\title{
Cognitive and Psychosocial Issues in Pediatric Multiple Sclerosis: Where We Are and Where We Need To Go
}

\author{
Maria Pia Amato ${ }^{1}$ \\ ${ }^{1}$ Department of Neurology, University of Florence, Florence, Italy \\ Neuropediatrics 2012;43:174-175.
}

Multiple sclerosis (MS) is a chronic demyelinating disease of the central nervous system in which cognitive dysfunction, major depression, and fatigue are common. While these problems are well documented in adults with MS, they are underestimated in children and adolescents with the disease that represent 3 to $5 \%$ of the whole MS population. ${ }^{1}$ Children and adolescents can be particularly vulnerable to MS-related cognitive issues as the disease occurs during key periods of age-expected brain growth, active primary myelination, and maturation of neural networks, moreover during the learning curve and key formative years in the academic career. It should also be considered that disease activity (clinical and magnetic resonance imaging [MRI]) is higher than in adults with $\mathrm{MS},{ }^{1}$ while, on the other hand, brain plasticity and ability for compensation is deemed to be more efficient in this age range. 1

In this issue of Neuropediatrics, Blaschek et $\mathrm{al}^{2}$ provide a comprehensive and insightful overview of available evidence on cognitive and psychosocial issues in the pediatric MS population and highlight the challenges and needs for future research in this field.

Similar to their adult counterparts, pediatric patients with MS are often cognitively impaired, with estimated figures of 30 up to $50 \%$ of the cases. ${ }^{1,3,4}$ At the same time, the review highlights a few unique features that specifically apply to the pediatric population. Beyond deficits of information processing speed, memory and executive functions that overlap with those described in adults with MS, one peculiar aspect in the pediatric population is involvement of language, usually spared in the adults. It is noteworthy that even mild linguistic deficits can have great functional impact and may negatively affect present and future academic achievements. Impact on general cognitive faculties and intelligence quotient has also been reported in children who are younger at the disease onset, suggesting a special vulnerability of this subgroup. 3,4 The study of large cohorts of patients is needed in the future to clarify whether the pattern of cognitive dysfunction in this population of patients may differ reflecting different developmental trajectories and depend on different classes of age and age at disease onset.

The review also highlights the apparent dissociation between the physical dimension and the cognitive dimension of MS-related disability in this patient population. As compared with adult patients, in fact, in children and adolescents, accrual of irreversible disability on the Expanded Disability Status Scale (EDSS) ${ }^{5}$ takes longer time. Cognitive dysfunction in children has been documented in close proximity with disease onset and may progress in the absence of any significant physical disability. ${ }^{1,4}$ Cognitive outcomes may represent a more relevant indicator of the disease in this population given their profound impact on academic performance and lifestyle. Although neurologists may not be directly involved in interventions for psychosocial issues, sensitivity to such issues is important so that appropriate referral for assessment and treatment can be made. Therefore, addressing cognitive issues beyond disease duration, relapses and physical disability appears to be of critical importance for patient counseling, rehabilitation, and therapeutic decision making. To this aim, there is a critical, unmet need for the development of valid and reliable assessment tools that can be used in everyday practice and research, with good norms that cover different classes of age.

As for psychosocial issues, fatigue is reported in 20 to $50 \%$ of the MS children, whereas the prevalence of depression varies from 6 to $46 \%{ }^{6,7}$ As in adults, fatigue and depression can interfere with the child's daily functioning and quality of life. Also in this case, systematic evaluation of fatigue and depression by means of validated instruments appears to be mandatory in routine follow-up care for all young patients.

Overall, the authors ${ }^{2}$ have provided an important additional dimension to our understanding of children with MS. Failing school performance is not simply due to physical disability or missed school days, but rather represents cognitive impairments that may worsen over time. While longterm studies are called for to assess the definitive cognitive
Address for correspondence and reprint requests Maria Pia Amato, M.D., Department of Neurosciences, University of Florence, Viale Morgagni, 85 50134 Florence, Italy (e-mail: mariapia.amato@unifi.it).
Copyright $\odot 2012$ by Thieme Medical Publishers, Inc., 333 Seventh Avenue, New York, NY 10001, USA. Tel: +1(212) 584-4662.
DOI http://dx.doi.org/ 10.1055/s-0032-1324400. ISSN 0174-304X. 
outcome in these subjects, early identification of the deficits is critical so that special accommodations can be implemented at home and at school.

Whether early initiation of MS therapies can help in improving cognitive function by confining the burden of the disease in the brain remains unclear and should also be addressed by future research. ${ }^{8}$ Finally, MRI research in this field is still in its early days. In particular, functional MRI studies may shed some light on adaptive and compensatory mechanisms and help measuring the effectiveness of therapeutic strategies based on cognitive rehabilitation.

\section{References}

1 Banwell B, Ghezzi A, Bar-Or A, Mikaeloff Y, Tardieu M. Multiple sclerosis in children: clinical diagnosis, therapeutic strategies, and future directions. Lancet Neurol 2007;6(10):887-902

2 Blaschek A, van's Gravesande KS, Heinen F, Pritsch M, Mall V, Calabrese P. Neuropsychological aspects of childhood multiple sclerosis: an overview. Neuropediatrics 2012; 43(4):176-183

3 Amato MP, Goretti B, Ghezzi A, et al; Multiple Sclerosis Study Group of the Italian Neurological Society. Cognitive and psychosocial features of childhood and juvenile MS. Neurology 2008; 70(20):1891-1897

4 Amato MP, Goretti B, Ghezzi A, et al; Multiple Sclerosis Study Group of the Italian Neurological Society. Cognitive and psychosocial features in childhood and juvenile MS: two-year follow-up. Neurology 2010;75(13):1134-1140

5 Kurtzke JF. Rating neurologic impairment in multiple sclerosis: an expanded disability status scale (EDSS). Neurology 1983;33 (11):1444-1452

6 MacAllister WS, Belman AL, Milazzo M, et al. Cognitive functioning in children and adolescents with multiple sclerosis. Neurology 2005;64(8):1422-1425

7 Goretti B, Portaccio E, Ghezzi A, et al; Multiple Sclerosis Study Group of the Italian Neurological Society. Fatigue and its relationships with cognitive functioning and depression in paediatric multiple sclerosis. Mult Scler 2012;18(3):329-334

8 Ghezzi A, Banwell B, Boyko A, et al. The management of multiple sclerosis in children: a European view. Mult Scler 2010;16 (10):1258-1267 